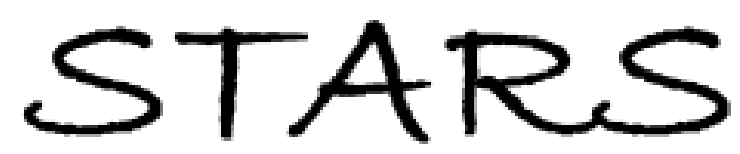

University of Central Florida

STARS

$1-1-2013$

\title{
A full-color reflective display using polymer-stabilized blue phase liquid crystal
}

Jin Yan

University of Central Florida

Shin-Tson Wu

University of Central Florida

Kung-Lung Cheng

Jyh-Wen Shiu

Find similar works at: https://stars.library.ucf.edu/facultybib2010 University of Central Florida Libraries http://library.ucf.edu

This Article is brought to you for free and open access by the Faculty Bibliography at STARS. It has been accepted for inclusion in Faculty Bibliography 2010 s by an authorized administrator of STARS. For more information, please contactSTARS@ucf.edu.

\section{Recommended Citation}

Yan, Jin; Wu, Shin-Tson; Cheng, Kung-Lung; and Shiu, Jyh-Wen, "A full-color reflective display using polymer-stabilized blue phase liquid crystal" (2013). Faculty Bibliography 2010 s. 4885.

https://stars.library.ucf.edu/facultybib2010/4885

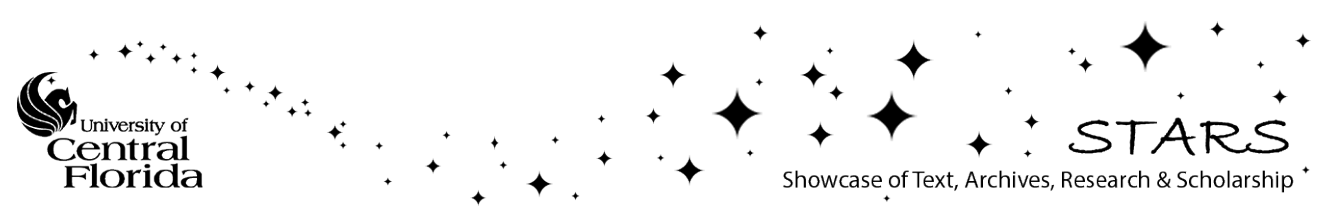




\section{A full-color reflective display using polymer- stabilized blue phase liquid crystal}

Cite as: Appl. Phys. Lett. 102, 081102 (2013); https://doi.org/10.1063/1.4793750

Submitted: 29 January 2013 . Accepted: 14 February 2013 . Published Online: 26 February 2013

Jin Yan, Shin-Tson Wu, Kung-Lung Cheng, and Jyh-Wen Shiu
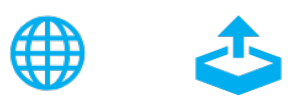

View Online

Export Citation

\section{ARTICLES YOU MAY BE INTERESTED IN}

Electric field-induced monodomain blue phase liquid crystals

Applied Physics Letters 102, 171110 (2013); https://doi.org/10.1063/1.4803922

Electro-optics of polymer-stabilized blue phase liquid crystal displays

Applied Physics Letters 94, 101104 (2009); https://doi.org/10.1063/1.3097355

Extended Kerr effect of polymer-stabilized blue-phase liquid crystals

Applied Physics Letters 96, 071105 (2010); https://doi.org/10.1063/1.3318288

\section{Applied Physics Reviews} Now accepting original research 


\title{
A full-color reflective display using polymer-stabilized blue phase liquid crystal
}

\author{
Jin Yan, ${ }^{1}$ Shin-Tson Wu, ${ }^{1, a)}$ Kung-Lung Cheng, ${ }^{2}$ and Jyh-Wen Shiu ${ }^{3}$ \\ ${ }^{1}$ CREOL, The College of Optics and Photonics, University of Central Florida, Orlando, Florida 32816, USA \\ ${ }^{2}$ Material and Chemical Research Laboratories, Industrial Technology Research Institute, Hsinchu, Taiwan \\ ${ }^{3}$ Display Technology Center, Industrial Technology Research Institute, Hsinchu, Taiwan
}

(Received 29 January 2013; accepted 14 February 2013; published online 26 February 2013)

\begin{abstract}
We demonstrate a full-color-capability reflective display using red, green, and blue sub-pixels of polymer-stabilized blue phase liquid crystal (BPLC) with different pitch lengths. Vivid colors originate from three-dimensional BPLC photonic crystalline structure. Surface alignment plays a key role to generate uniform and saturated colors in the voltage-off state. Analogous grayscale is achieved by the electric-field-induced unwinding of double-twist structure. This working principle is drastically different from the phase retardation effect of conventional liquid crystal displays. Moreover, the submillisecond response time enables crisp video displays without image blurring. Potential applications for reflective 3D display are also analyzed. (C) 2013 American Institute of Physics. [http://dx.doi.org/10.1063/1.4793750]
\end{abstract}

A direct-view reflective display uses ambient light to readout the displayed images. ${ }^{1}$ It offers several advantages, such as good sunlight readability, low power consumption, and no need for a polarizer. In the past five decades, several types of reflective displays have been developed, such as twisted-nematic (TN) liquid crystal wrist watch, ${ }^{2}$ mixedmode twisted nematic for Gameboy Color, ${ }^{3}$ cholesteric liquid crystal display for electronic paper, ${ }^{4,5}$ electrowetting display, ${ }^{6}$ electrofluidic display, ${ }^{6}$ and electrophoretic display. ${ }^{6}$ The major challenges of most reflective displays are unsaturated color, insufficiently fast response time to display videos, difficult to achieve natural grayscales for bistable devices, and relatively low contrast ratio due to surface reflection.

In this paper, we propose a reflective display using polymer-stabilized blue phase liquid crystal (PS-BPLC). ${ }^{7-11}$ It exhibits several attractive features, such as no need for polarizer and color filters, relatively narrow reflection band, submillisecond response time, and analogous grayscales by controlling the applied voltage.

Blue phases appear between chiral-nematic phase and isotropic phase in a highly twisted chiral-nematic liquid crystal. ${ }^{5}$ They exhibit self-assembled cubic structures, and the local refractive index variation results in selective Bragg reflections in the spectral range. The reflection wavelength can be expressed as ${ }^{12}$

$$
\lambda=\frac{2 n a}{\sqrt{h^{2}+k^{2}+l^{2}}},
$$

where $n$ and $a$ denote average refractive index and lattice constant of blue phases, respectively, and $h, k$, and $l$ are the Miller indices of a crystal plane. For transmissive displays, ${ }^{13-16}$ the reflection bands should be shifted to ultraviolet region by employing a high concentration chiral dopant so that it is optically isotropic in the visible spectral region.

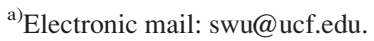

However for reflective displays, we intentionally adjust the pitch lengths to obtain reflective colors in the visible region. Since blue phases have delicate and fragile structures, the lattices of the cubic structures can be deformed by an electric field, which leads to a shift of the reflection wavelength. Based on the lattice deformation, electrically tunable colors using BPLCs have been demonstrated. ${ }^{17,18}$ However for polymer-stabilized blue phases, the cubic structures are stabilized by polymer network so that the lattice deformation can only take place at a relatively high field. ${ }^{19}$ In the low field region, the applied electric field mainly reorients the liquid crystal (LC) molecules but not deform the lattices.

Figure 1 illustrates the device structure and operation principles of the reflective BPLC display. Each pixel consists of three sub-pixels which reflect red, green, and blue colors, respectively. The bright state is achieved in the voltage-off state, as shown in Fig. 1(a). Here, the sub-pixels are separated by striped wall structures. ${ }^{20}$ Another way to obtain full color is to stack red, green, and blue (RGB) layers, similar to a cholesteric display. ${ }^{21}$ As voltage increases, the LC molecules tend

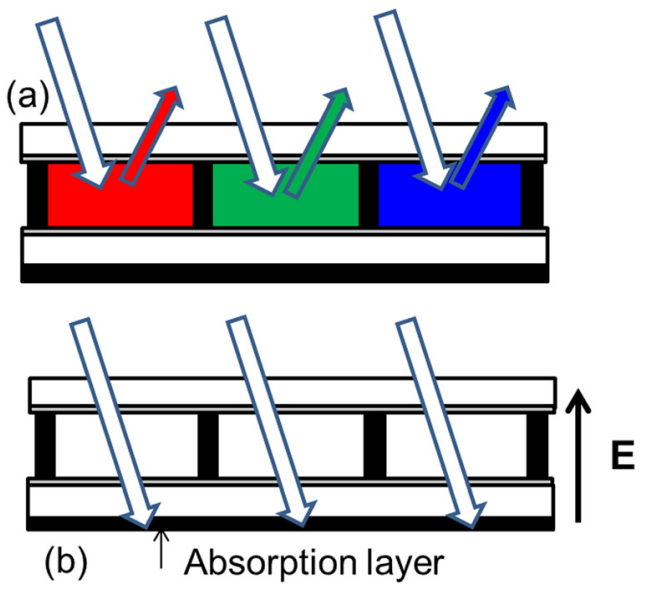

FIG. 1. Working principle of the proposed reflective display: (a) bright state at $\mathrm{V}=0$, and (b) black state at $\mathrm{V}>0$. To obtain narrow band reflection, the BPLC cell has surface alignment layers. 
to align with the electric field because the employed LC has a positive dielectric anisotropy. As a result, the local refractive index difference is reduced, and the reflectance gradually decreases. The transmitted light is absorbed by the painted absorption layer on the substrate to achieve dark state, as Fig. 1(b) shows.

To prove concept of the proposed reflective display, we first prepared a BPLC sample which reflects green color. To study the effect of surface alignment, the BPLC precursor was injected into three cells: (1) cell-1 made of two ITO glass substrates without polyimide (no PI); (2) a TN cell with polyimide and orthogonal rubbing on top and bottom substrates; (3) a homogenous (HG) cell with antiparallel rubbing on the two substrates. The cell gaps for the three cells were $\sim 5 \mu \mathrm{m}$. UV irradiation was performed near the transition temperature from blue phase to chiral-nematic phase during cooling process. The light intensity was $2 \mathrm{~mW} / \mathrm{cm}^{2}$, and the curing time was $30 \mathrm{~min}$. After polymerization, the blue phase composites were stabilized.

Figure 2(a) shows the reflective photo of cell-1 taken under an optical microscope with crossed polarizers. Without alignment, the lattices have different orientations so that the platelets can be observed. Figure 2(b) shows the photo of the TN cell. Different from cell-1, this cell exhibits uniform texture, because the surface alignment provides anchoring energy to confine the orientation of lattices. The HG cell has a similar texture to the TN cell; the photo is not shown here. Figure 2(c) depicts the transmittance and reflectance for the three cells. The baseline reflection is due to the surface and ITO reflections. The cell without PI shows a very small reflection band at $\sim 560 \mathrm{~nm}$, and the transmittance
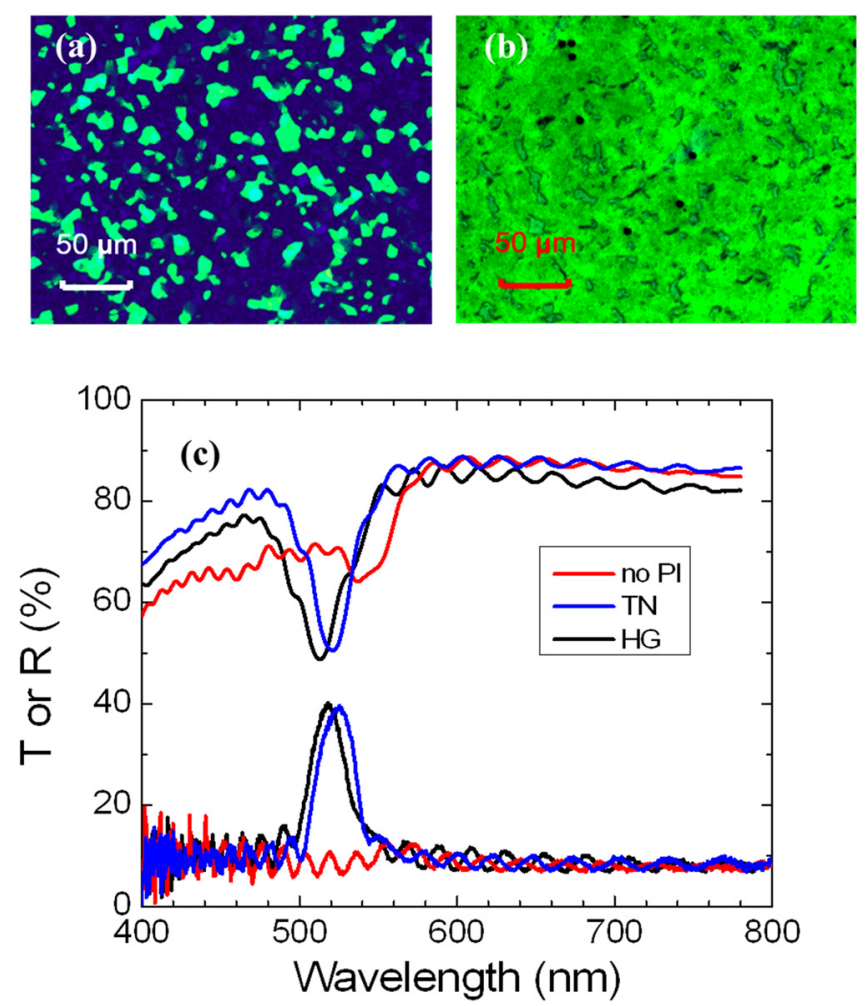

FIG. 2. Reflective photo of (a) PS-BPLC cell-1 without polyimide and (b) TN cell; (c) measured transmittance and reflectance of three cells with different surface alignments. decreases dramatically for wavelengths shorter than $560 \mathrm{~nm}$; green-bluish colors can be observed. From the measured spectra, we consider that the observed colors are originated from both reflection and scattering, because the blue phase has multiple domains when there is no surface alignment. Both TN and HG cells show a clear and fairly narrow reflection band at $\sim 520 \mathrm{~nm}$; the full width at half maximum is $\sim 25 \mathrm{~nm}$. Similar performances of $\mathrm{TN}$ and $\mathrm{HG}$ cells are expected since the cell gap is much larger than the lattice constant of the blue phase. Therefore, the alignment direction is not critical in the proposed reflective display as long as there is surface alignment. The scattering at shorter wavelengths is much weaker than that of the cell without PI. Thus, the observed color mainly comes from Bragg reflection, and the color is more saturated.

In the following, we choose $\mathrm{TN}$ cells for further investigating its electro-optic performance because our TN cells have uniform cell gap. We prepared three TN PS-BPLC cells that reflect blue, green, and red colors, respectively. The employed material consists of nematic LC host HTG-135200 (HCCH, China), chiral dopant with HTP $\approx 126 / \mu \mathrm{m}(\mathrm{HCCH}$, China), 10 wt. \% monomers [6 wt.\% RM257 (Merck) and 4 wt. \% TMPTA (1,1,1-Trimethylolpropane Triacrylate, Sigma Aldrich)], and 0.6 wt. $\%$ photoinitiator. The concentrations of LC host and chiral dopant are adjusted to achieve different pitch lengths. Our nematic LC host has the following physical properties: $\Delta \mathrm{n}=0.2$ at $\lambda=633 \mathrm{~nm}, \Delta \varepsilon=96$ at $1 \mathrm{kHz}$, and $22^{\circ} \mathrm{C}$, and clearing temperature $\mathrm{T}_{\mathrm{c}}=97^{\circ} \mathrm{C}$. The cell gaps are $\sim 5 \mu \mathrm{m}$.

Figure 3 depicts the measured reflectance spectra of the three cells under different voltages. The insets show reflective photos under microscope with crossed polarizers. The three cells are laminated with anti-reflection films so that surface reflection from the interface between the glass and air is reduced to $\sim 1 \%$. The baselines are the interface reflections due to refractive index mismatch between ITO electrodes and glass substrates. All the reflection curves are normalized to that of a mirror. From Fig. 3, all the reflectance spectra exhibit a relatively narrow bandwidth which indicates saturated colors. The blue and green cells show a reflectance over $35 \%$, but the red cell has a slightly lower reflectance $(26 \%)$ because of its longer pitch length, which leads to fewer periods within the $5-\mu \mathrm{m}$ cell gap. The reflectance can be improved by employing a thicker cell gap, but the tradeoff is increased voltage. As the voltage increases, LC molecules are reoriented along the electric field, and the double twist structures are gradually unwound, so the reflectance decreases. When most of the LC molecules are reoriented by the electric field, the cell becomes transparent, and the reflectance touches the baseline. Therefore, natural grayscales are achieved. On the other hand, the lattices are stabilized by the polymer network, so the resonance wavelengths hardly shift. The small blue shifts are due to the electric-field-induced refractive index change.

In Fig. 3, the peak wavelength of the green cell was intentionally adjusted to match an Argon-ion laser line at $\lambda=514 \mathrm{~nm}$. We choose the green cell to further study the voltage-dependent reflectance (VR) behavior. The light incident on the BPLC sample is left-handed circularly polarized by combining a linear polarizer and a $\lambda / 4$ plate. The incident 

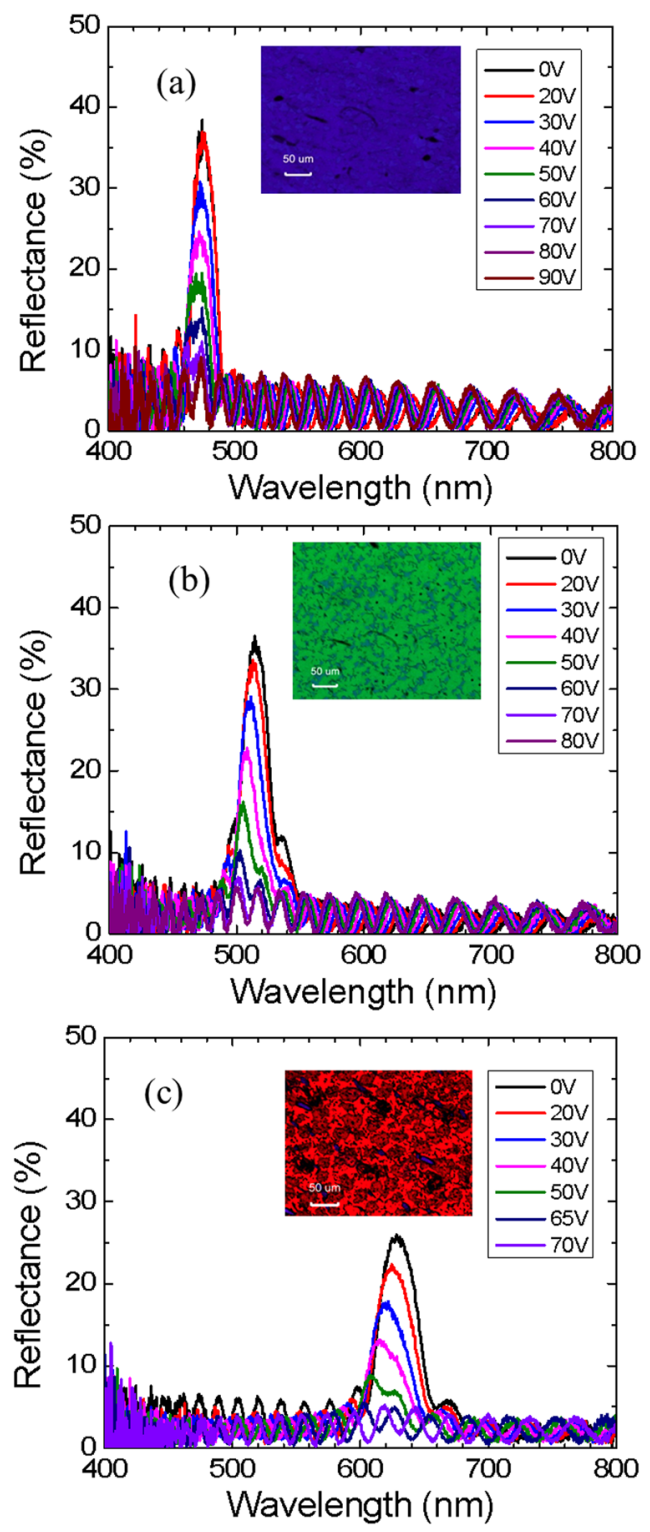

FIG. 3. Voltage-dependent reflection spectrum of (a) blue cell, peak wavelength $\lambda_{0}=477 \mathrm{~nm}$; (b) green cell, $\lambda_{0}=514 \mathrm{~nm}$; (c) red cell, $\lambda_{0}=634 \mathrm{~nm}$. The insets are reflective microscope photos with crossed polarizers.

angle is $\sim 5^{\circ}$. If we rotate the $\lambda / 4$ plate by $90^{\circ}$, the polarization state of the incident light would become right-handed circular polarization, and the reflectance would become very low. Therefore, the BPLC cell reflects left-handed circularly polarized light, which is consistent with the handedness of the employed chiral dopant.

Figure 4 depicts the measured VR curves for the green cell with a left-handed circularly polarized (LCP) light and a linearly polarized (LP) light by removing the $\lambda / 4$ plate. The reflectance is normalized to that of a mirror. With LCP light, the reflectance is expected to be $2 \mathrm{X}$ of the LP light, but the measured ratio is only $\sim 1.5 \mathrm{X}$. The reason is that the reflected light is not perfectly circularly polarized. The contrast ratio is $\sim 17: 1$ for the LP light and $\sim 15: 1$ for the LCP light at a voltage of $70 \mathrm{~V}$. Hysteresis is related to the BPLC material and peak electric field. ${ }^{22,23}$ When the cell is driven at $70 \mathrm{~V}$, the hysteresis is $\sim 9 \%\left(\Delta \mathrm{V} / \mathrm{V}_{\max }, \Delta \mathrm{V}\right.$ is the voltage difference at $50 \%$ reflectance and $\mathrm{V}_{\max }$ is the maximum driving voltage). A smaller hysteresis is obtained if we limit $\mathrm{V}_{\max }$ to

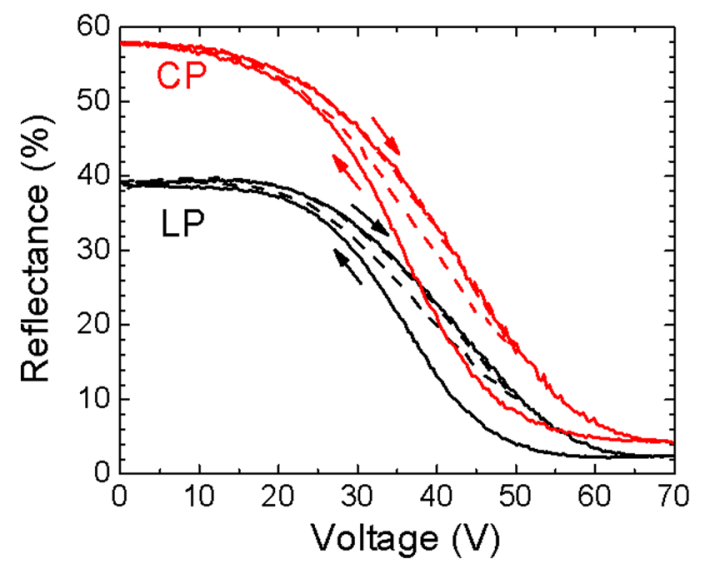

FIG. 4. Measured VR curve of the green cell with left-handed circularly polarized (CP) and LP incident light.

$50 \mathrm{~V}$, but the tradeoff is lower contrast ratio. We also measured the response time of the reflective BPLC cell at room temperature. The rise time is $\sim 75 \mu \mathrm{s}$ and decay time $\sim 436 \mu$ s with an applied voltage of $70 \mathrm{~V}$ and $f=1 \mathrm{kHz}$.

It has been reported that the light reflected by blue phases could be circularly or partially circularly polarized. $^{24-28}$ In our case, we experimentally investigated the polarization state of the reflected light. An analyzer was placed before the detector to examine the polarization state. We measured the reflected intensity at $360^{\circ}$ circle with a LCP light and then calculated the polarization state accordingly. Results are plotted by the solid red line in Fig. 5. The reflected light is elliptically polarized, with an axial ratio of $\sim 0.68$. Next, we repeated the same experiment with a linearly polarized light by removing the $\lambda / 4$ plate. The polarization state of the reflected light is shown as dashed blue lines in Fig. 5 . The axial ratio is $\sim 0.55$. In both cases, the reflected light is left-handed elliptically polarized. For an unpolarized incident light, the reflectance is similar to that of linearly incident light since the unpolarized light can also be decomposed into a left-handed and a right-handed circular polarization. The reflected light is also left-handed elliptically polarized (not shown here). To explain this phenomenon, we

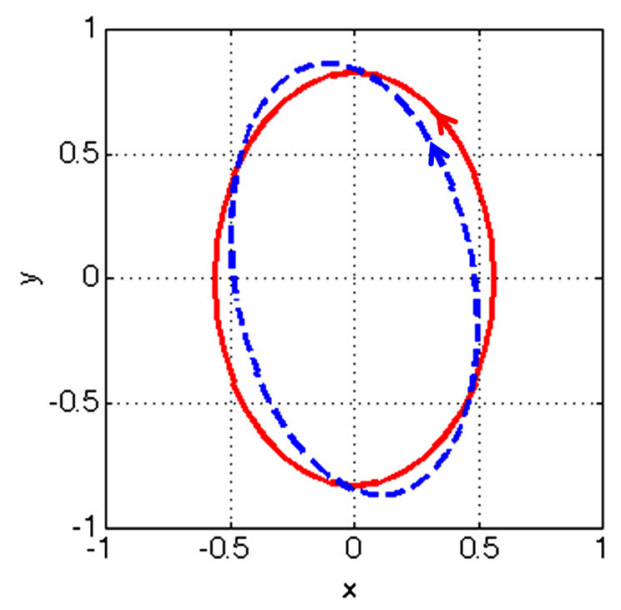

FIG. 5. Calculated polarization state of reflected light through measured data for a circularly polarized incident light (solid red line) and a linearly polarized light (blue dashed lines). $x$ represents the absorption axis of the polarizer. 


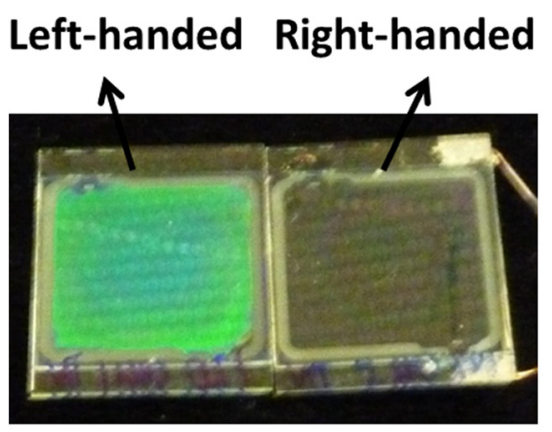

(a)

\section{Left-handed Right-handed}

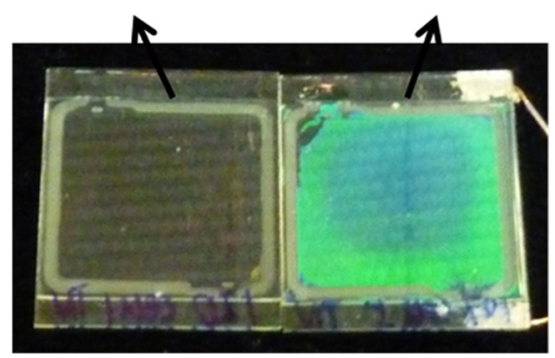

(b)

FIG. 6. Concept of proposed 3D reflective display: the left pixel employs a left-handed chiral dopant and the right pixel employs a right-handed chiral dopant. (a) Photo taken with a left-handed circular polarizer, and (b) photo taken with a right-handed circular polarizer.

need to know that the reflected light is a superposition of three components: (1) Bragg reflection from the BPLC, (2) interface reflections, and (3) the alignment layers can also affect the LC molecular orientation which would further change the polarization state. Thus, the reflected light cannot be a perfect circular polarization; it favors left-handed circular polarization provided that a left-handed chiral dopant is employed.

Due to the property of selective reflection of polarization, the proposed reflective display has potential application in 3D displays. One color pixel can be divided into two subpixels, which employs left-handed chiral dopant and righthanded chiral dopant, respectively. Figure 6 shows two adjacent sub-pixels, wherein the left pixel employs left-handed chiral dopant and the right pixel employs right-handed chiral dopant. Therefore, the left pixel reflects left-handed circularly polarized light, while the right pixel reflects righthanded circular light. Circularly polarized eyeglasses are required for this 3D display. For the left eye wearing lefthanded circular polarizer, it sees the reflection from the left pixel (Fig. 6(a)). Similarly, for the right eye wearing righthanded circular polarizer, it sees the reflection from the right pixel (Fig. 6(b)). With absorptive layer on the bottom substrate and anti-reflection coating on the top substrate, the right pixel would look dark for the left eye and the left pixel would look dark for the right eye. Therefore, the left eye can only see the left pixel which displays left-eye image, and the right eye can only see the right pixel which displays right-eye image. In this way, we will be able to observe $3 \mathrm{D}$ images. In Fig. 6, the fringes and the color difference between the center and edge of the cells are due to the cell
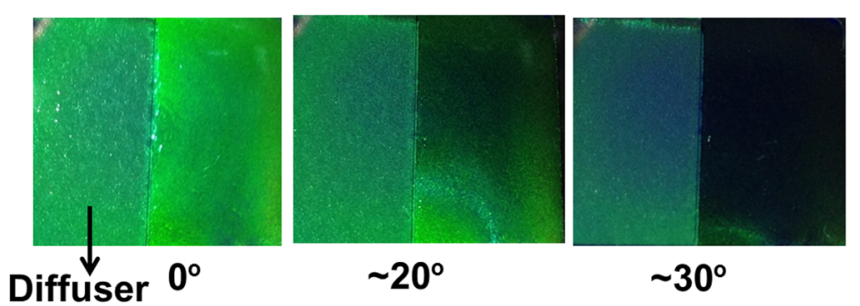

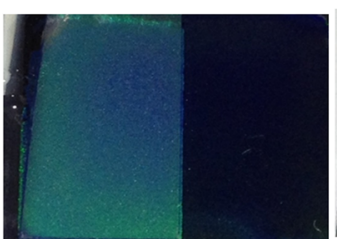

$\sim 4^{\circ}$

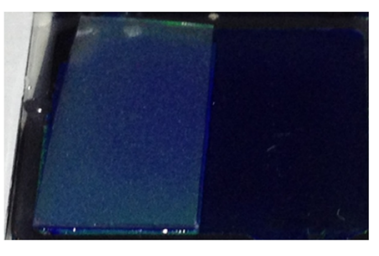

$\sim 60^{\circ}$
FIG. 7. Viewing angle of the reflective display. Diffuser was laminated on top of the left side of the cell.

gap variations across the cell. The cell gap should satisfy $d=N \times a$, where $\mathrm{N}$ is an integer number and $a$ is the lattice constant. Therefore, the cell gap variations could make the reflection wavelength slightly different.

We also investigated the viewing angle of the proposed reflective display. Because blue phase has photonic crystalline structure, the intrinsic viewing angle is narrow. To widen the viewing angle, we laminated a diffuser on top of the left part of the cell for comparison, as Fig. 7 depicts. The light source was placed in the normal direction. When viewing at normal direction $\left(0^{\circ}\right)$, we can observe vivid green color on both pixels. The left part has a slightly lower reflectance because part of the reflected light is diffused to other directions. However, as the viewing angle gets larger than $20^{\circ}$, the right pixel appears dark blue while the left pixel remains visible up to $60^{\circ}$, although a significant color shift occurs. In practical applications, the viewing angle should be wider because the ambient light impinges from all the directions.

In conclusion, we have demonstrated a reflective display using polymer-stabilized blue phase liquid crystals. Such a vivid-color reflective display is polarizer-free and color-filter-free, which gives potential for flexible displays. It shows narrow reflection bandwidth, submillisecond response time, and natural grayscales. It also exhibits potential for 3D applications. The proposed display opens a gateway for 3D reflective displays and will make significant impacts to display industry.

The authors are indebted to Daming $\mathrm{Xu}$, Zhenyue Luo, Yuan Chen, Su Xu, and Honghua Hu for technical assistance and discussion and Industrial Technology Research Institute (ITRI, Taiwan) for financial support.

${ }^{1}$ S. T. Wu and D. K. Yang, Reflective Liquid Crystal Displays (Wiley, New York, 2001).

${ }^{2}$ M. Schadt and W. Helfrich, Appl. Phys. Lett. 18, 127 (1971).

${ }^{3}$ S. T. Wu and C. S. Wu, Appl. Phys. Lett. 68, 1455 (1996).

${ }^{4}$ D. K. Yang, J. W. Doane, Z. Yaniv, and J. Glasser, Appl. Phys. Lett. 64, 1905 (1994).

${ }^{5}$ H. S. Kitzerow and C. Bahr, Chirality in Liquid Crystals (Springer, New York, 2001).

${ }^{6}$ J. Heikenfeld, P. Drzaic, J. S. Yeo, and T. Koch, J. Soc. Inf. Disp. 19, 129 (2011). 
${ }^{7}$ H. Kikuchi, M. Yokota, Y. Hisakado, H. Yang, and T. Kajiyama, Nat. Mater. 1, 64 (2002).

${ }^{8}$ Y. Hisakado, H. Kikuchi, T. Nagamura, and T. Kajiyama, Adv. Mater. 17, 96 (2005)

${ }^{9}$ J. Yan, L. Rao, M. Jiao, Y. Li, H. C. Cheng, and S. T. Wu, J. Mater. Chem. 21, 7870 (2011).

${ }^{10}$ J. Yan and S. T. Wu, Opt. Mater. Express 1, 1527 (2011).

${ }^{11}$ H. Kikuchi, Liquid Crystalline Blue Phases (Springer, Berlin/Heidelberg, 2008), p. 106.

${ }^{12}$ Z. Ge, S. Gauza, M. Jiao, H. Xianyu, and S. T. Wu, Appl. Phys. Lett. 94, 101104 (2009).

${ }^{13}$ L. Rao, Z. Ge, S. T. Wu, and S. H. Lee, Appl. Phys. Lett. 95, 231101 (2009).

${ }^{14}$ L. Rao, J. Yan, S. T. Wu, S. Yamamoto, and Y. Haseba, Appl. Phys. Lett. 98, 081109 (2011).

${ }^{15}$ H. C. Cheng, J. Yan, T. Ishinabe, and S. T. Wu, Appl. Phys. Lett. 98, 261102 (2011).

${ }^{16}$ M. Wittek, N. Tanaka, D. Wilkes, M. Bremer, D. Pauluth, J. Canisius, A. Yeh, R. Yan, K. Skjonnemand, and M. K. Memmer, SID Int. Symp. Digest Tech. Papers 43, 25 (2012).

${ }^{17}$ H. S. Kitzerow, Mol. Cryst. Liq. Cryst. 202, 51 (1991).
${ }^{18}$ G. Heppke, M. Krumrey, and F. Oestreicher, Mol. Cryst. Liq. Cryst. 99, 99 (1983).

${ }^{19}$ S. Y. Lu and L. C. Chien, Opt. Lett. 35, 562 (2010).

${ }^{20}$ C. L. Chin, K. L. Cheng, Y. R. Wang, C. C. Tsai, and J. W. Shiu, SID Int. Symp. Digest Tech. Papers 43, 548 (2012).

${ }^{21}$ M. Okada, T. Hatano, and K. Hashimoto, SID Int. Symp. Digest Tech. Papers 28, 1019 (1997).

${ }^{22}$ K. M. Chen, S. Gauza, H. Xianyu, and S. T. Wu, J. Disp. Technol. 6, 318 (2010).

${ }^{23}$ L. Rao, J. Yan, S. T. Wu, Y. C. Lai, Y. H. Chiu, H. Y. Chen, C. C. Liang, C. M. Wu, P. J. Hsieh, S. H. Liu, and K. L. Cheng, J. Disp. Technol. 7, 627 (2011).

${ }^{24}$ R. M. Hornreich and S. Shtrikman, Phys. Rev. A 28, 1791 (1983).

${ }^{25}$ C. Bohley and T. Scharf, J. Opt. A: Pure Appl. Opt. 6, S77 (2004).

${ }^{26}$ S. Yokoyama, S. Mashiko, H. Kikuchi, K. Uchida, and T. Nagamura, Adv. Mater. 18, 48 (2006).

${ }^{27}$ F. Castles, F. V. Day, S. M. Morris, D. H. Ko, D. J. Gardiner, M. M. Qasim, S. Nosheen, P. J. W. Hands, S. S. Choi, R. H. Friend, and H. J. Coles, Nat. Mater. 11, 599 (2012).

${ }^{28}$ J. Yan, Z. Luo, S. T. Wu, J. W. Shiu, Y. C. Lai, K. L. Cheng, S. H. Liu, P. J. Hsieh, and Y. C. Tsai, Appl. Phys. Lett. 102, 011113 (2013). 